\title{
Pesticide-induced Histopathological Changes in the Freshwater Fishes of Kuttanand, Kerala-A Tool to Assess Water Quality and the Health Status of Fishes
}

\author{
B.T. SULEKHA ${ }^{1}$ and T.V. ANNA \\ MERCY $^{2 *}$ \\ ${ }^{1}$ S.N.College, Quilon, Kerala, India \\ ${ }^{2}$ College of Fisheries, Kerala Agricultural University, Panangad, Cochin 682 506, \\ Kerala, India.
}

\begin{abstract}
Kuttanad, the rice bowl of Kerala, is a region where overdose application of pesticide is prevalent during the punja cultivation periods. According to the data compiled by Kuttanad Water Balance Study Project, 485 tonnes of pesticides were applied in Kuttanad on an annual basis of which 370 tonnes were used for the punja crop alone (KWBSP, 1990). In such a degraded aquatic environment, particularly where pollutants occur at chronic sublethal concentrations, changes in the structure and functions of aquatic organisms occur more frequently than their mass mortality. Therefore, one of the possible methods of assessing the effects of pollutants on fresh water fish inhabiting this ecosystem is to examine their organs for morphological changes. In fishes, apart from lethal effects of pesticides and the consequent mortality of eggs, larvae, and adults, their prolonged exposure in sublethal concentration may also result in reproductive abnormality, stock recruitment, deformities of eggs and larvae, retardation of hatchling percentage and body abnormalities. In the present study, a tool developed by Bernet et al. (1999) is used to assess the histopathological conditions; hence, histopathology is used as a tool to assess the health status of two freshwater fishes of Kuttanad, viz., Etroplus suratensis and Anabas testudineus. The organ index calculated based on various reaction patterns of the different organs of fishes exposed to sublethal concentrations of monocrotophos for a period of 30 days showed that gills were severely affected, liver was moderately affected, and kidney was the mildly affected organ, irrespective of fish species. Histopathology provides evidences of adaptation to degeneration, and histopathological alterations can be used as biomarkers of environmental pollution by organic chemicals. Histological changes in fish gill should become a rapid "early warning system" for water quality assessment in sublethal and chronic situations, as the toxicants induce changes at lower levels of biological organization prior to organismic changes.
\end{abstract}

\section{Introduction}

Kuttanad, the rice bowl of Kerala, India, is a region where overdose application of pesticide is prevalent during the punja cultivation periods. Traditionally, there are three cropping seasons for paddy in Kerala, the virippu, mundakan, and punja seasons. Punja crop, the traditional crop of Kuttanad, is sown in November to December and the

*Corresponding Author.

Email : annamercy2002@yahoo.co.in 
harvest takes place by the end of March. The peak period of the pest damage, particularly by the brown plant hopper, is from February to March, which not only reduces the yield but also entails additional expenses for pesticides. There is no systematic crop surveillance and therefore, farmers arbitrarily apply pesticides at regular intervals. These ways of treatments are ineffective as well as unwanted and can cause severe damage to Kuttanad ecosystem. (KWBSP 1990).

According to the data compiled by Kuttanad Water Balance Study Project, 485 tonnes of pesticides were applied in Kuttanad on an annual basis of which 370 tonnes were used for the puncha crop alone (KWBSP 1990). Dimecron, Monocrotophos, Henosan, Thymet, Fernoxan, and Nuvacron are the major components of the pesticides being used in Kuttanad. In such a degraded aquatic environment, particularly where pollutants occur at chronic sublethal concentrations, changes in the structure and functions of aquatic organisms occur more frequently than their mass mortality. Therefore, one of the possible methods of assessing the effects of pollutants on fresh water fish inhabiting this ecosystem is to examine their organs for morphological changes.

In fishes, apart from lethal effects of pesticides and the consequent mortality of eggs, larvae, and adults, their prolonged exposure in sublethal concentration may also result in reproductive abnormality, stock recruitment, deformities of eggs and larvae, retardation of hatchling percentage, and body abnormalities. Evidence of retardation of natural propagation of fishes is already discernible in Kuttanad due to very low yield registered from these regions (Kurup et al. 1990). Hence, water pollution can lead to different changes, ranging from biochemical alterations in single cell into changes in whole populations. In general, the end points used in toxicity studies are mortality, survival, and growth with acute toxicity tests. These parameters are quite appropriate, but for long-term sublethal concentrations, these relevant parameters are difficult to ascertain. In the past, there were no tools to measure the magnitude of histopathological conditions in the affected organs. However, at present, many tools are available. Hence, in the present study, histopathological parameters are used to assess the nature and magnitude of toxic effects of pesticides that are being widely applied in the paddy fields of Kuttnad. This analysis is "user friendly" for the field investigator (Hinton 1993).

The advantage of histopathology as a biomarker lies in its intermediate location with regard to the level of biological organization (Adams et al. 1989). Histological changes appear as a medium-term response to sublethal stressors, and histology provides a rapid method to detect the effects of irritants, especially chronic ones in various tissues and organs (John et al. 1993). Histopathological analysis yields data on a number of organs and permits localization of lesions within specific cell types. With a thorough prior knowledge of normal anatomy, the investigator can use histological analysis to detect alterations in tissues and organs caused by exposure to toxicants. When concentration of a toxicant is sufficient to result only in cellular injury and not death, sublethal (adaptive) changes can be observed in affected cells. 
The exposure of fish to chemical contaminants is likely to induce a number of lesions in body organs like gills, liver, and kidney. These organs are suitable for histological examination to determine the effect of extent of pollution (Hinton 1993). Gills exhibit large surfaces, which are subjected to direct and permanent contact with potential irritants. Liver plays a key role in metabolism and subsequent excretion of xenobiotics and is also the site of vitellogenin production. Kidney is important for the maintenance of a stable internal environment and partially involved in the metabolism of xenobiotics (Hinton 1993).

In the above-mentioned conditions, it is felt that a study on the pesticide-induced histopathological changes in selected fishes would be helpful in bringing out the lethal effect caused to fish health due to ubiquitous application of pesticides and henceforth establishing the necessity for a judicious use of pesticides in agriculture in future.

\section{Materials and methods}

Juveniles of Etroplus maculatus (E. maculatus) and Anabas testudineus (A. testudineus) were collected from pollution-free ponds from the natural habitat. These fishes (size $47.5 \pm 9.0 \mathrm{~mm}$ and $71.5 \pm 6.0 \mathrm{~mm}$ in total length and $330 \pm 80 \mathrm{mg}$ and 750 $\pm 150 \mathrm{mg}$ in weight, respectively) were acclimatized to the laboratory conditions for 14 days prior to the bioassay. During these periods, they were fed ad libitum once a day on fresh clam meat. The experiments on the lethal and sublethal toxicity of monocrotophosan organophosphate pesticide-on the juveniles of E. maculatus and A. testudineus, the true denizens of Kuttanad paddy fields, were conducted for 48 hours and 30 days, respectively, during the period of investigation.

Based on the $\mathrm{LC}_{50}$ values (Mercy et al. 2000), five nominal concentrations of the pesticide were selected for sublethal toxicity studies. Maximum and minimum sublethal concentrations were chosen based on Konar (1969) and Sprague (1973). The experimental fishes were exposed to such sublethal concentrations for a period of 30 days. The concentrations of pesticides used for each sublethal exposure are given in Table 1.

Table1. Forty-eight-hour $\mathrm{LC}_{50}$ values and sublethal concentrations chosen for the experiment

\begin{tabular}{lllllllll}
\hline Fish species & Pesticide & \multicolumn{6}{c}{ 48-hr.LC } & \\
50 & $(\mathrm{ppm})$ & \multicolumn{5}{c}{ Sublethal concentrations $\left(\mathrm{mg} . \mathrm{L}^{-1}\right)$} \\
\hline Etroplus maculatus Monocrotophos & 3.36 & 0.0 & 0.1 & 0.3 & 0.6 & 1.0 & 1.5 \\
Anabas testudineus & 102.59 & 0.0 & 2.0 & 5.0 & 10.0 & 18.0 & 36.0 \\
\hline
\end{tabular}


Sublethal exposure was carried out in a static system where water and pesticide medium were renewed every 24 hours to obtain the desired pesticide concentration. A control free of pesticide was also maintained in each experiment. All the treatments were made in triplicates. Ten healthy fishes, each of the target species, chosen at random from the acclimated stock were reared in 32 litres of water in seasoned cement cisterns. The ratio of the animal wet-weight to water volume ranged from 0.4899 to 2.7875 gm.L${ }^{1}$. The tanks were covered with plastic mesh nets to prevent the escape of the fishes by jumping. All the experiments were conducted in ambient temperature $\left(28 \pm 2^{\circ} \mathrm{C}\right)$. The dissolved oxygen, $\mathrm{pH}$, and temperature in the different treatments were measured immediately before and after the pesticide inoculation. After 30 days, i.e. after the termination of the experiments, five specimens from each of the treated as well as the control group were killed and the target organs such as the gills, liver, and kidney were dissected out and fixed immediately in Bouin's fluid. Histological sections were prepared based on standard procedures and stained using hematoxylin and eosin. Each organ is observed for its detailed histology. Same species of fishes were collected from the paddy fields of Kuttanad during the months of February and March, and histological preparations were carried out for the target organs and were observed for their histopathological lesions.

In the present study, histopathological conditions of different organs were assessed based on Bernet et al. (1999) who classified the histopathological changes of each organ into five reaction patterns (Table 2). Each pattern includes several alterations with respect to either functional unit of the organ or entire organ. Calculation of the index values was based on an importance factor $(w)$ and score value $(a)$.

\section{Importance factor $(w)$}

The relevance of a lesion depends on its pathological importance, i.e. how it affects organ function and the ability of the fish to survive. This is taken into account by an importance factor assigned to every alteration listed in the histological description.

The alterations are classified into three important factors:

1) Minimal pathological importance, the lesion is easily reversible as exposure to irritants ends; 2) Moderate pathological importance, the lesion is reversible in most cases if the stressor is neutralized; and 3) Marked pathological importance, the lesion is generally irreversible, leading to partial or total loss of the organ function.

Score value (a) Every alteration is assessed using a score ranging from 0 to 6 , depending on the degree and extent of alteration: (0) unchanged; (2) mild occurrence; (4) moderate occurrence; and (6) severe occurrence (diffuse lesion). Intermediate values were also considered. 
Table 2. Histopathological assessment tools for 3 fish organs (i.e. gills, liver, and kidney). An importance factor $\left(\mathrm{W}_{\text {org rp alt }}\right)$ ranging from 1 to 3 is assigned to every alteration: it is composed of the respective organ (org), the reaction pattern (rp), and the alteration (alt)*. \# Extracted from Bernet et al. (1999)

\begin{tabular}{|c|c|c|c|c|c|}
\hline Reaction pattern & $\begin{array}{l}\text { Functional unit } \\
\text { of the tissue }\end{array}$ & Alteration \# & $\begin{array}{l}\text { Importance } \\
\text { factor }\end{array}$ & $\begin{array}{l}\text { Score } \\
\text { value }\end{array}$ & Index \\
\hline \multicolumn{2}{|l|}{ Gills } & \multicolumn{4}{|l|}{ Hemorrhage/hyperemia } \\
\hline \multirow{2}{*}{\multicolumn{2}{|c|}{ Circulatory disturbances }} & /aneurysm & $\mathrm{WGC} 1=1$ & $\mathrm{aGC1}$ & IGC \\
\hline & & Intercellular edema & $\mathrm{WGC} 2=1$ & $\mathrm{aGC} 2$ & \\
\hline \multirow[t]{14}{*}{ Regressive changes } & \multirow[t]{8}{*}{ Epithelium } & Architectural and structural & & & \multirow{14}{*}{ IGR } \\
\hline & & alterations & WGR1 = 1 & aGR1 & \\
\hline & & Plasma alterations & WGR2 = 1 & aGR2 & \\
\hline & & Deposits & WGR3 = 1 & aGR3 & \\
\hline & & Nuclear alterations & WGR4 = 2 & aGR4 & \\
\hline & & Atrophy & WGR5 = 2 & aGR5 & \\
\hline & & Necrosis & WGR6 = 3 & aGR6 & \\
\hline & & Rupture of the pillar cells & & & \\
\hline & \multirow[t]{6}{*}{ Supporting tissue } & \multicolumn{3}{|l|}{ Architectural and structural } & \\
\hline & & Plasma alterations & WGR8 = 1 & aGR8 & \\
\hline & & Deposits & WGR9 = 1 & aGR9 & \\
\hline & & Nuclear alterations & WGR $10=2$ & aGR10 & \\
\hline & & Atrophy & WGR11 = 2 & aGR11 & \\
\hline & & Necrosis & $\mathrm{WGR} 12=3$ & aGR12 & \\
\hline \multicolumn{6}{|l|}{ Progressive } \\
\hline \multirow[t]{4}{*}{ changes } & \multirow[t]{2}{*}{ Epithelium } & Hypertrophy & WGP1 = 1 & aGP1 & \multirow[t]{4}{*}{ IGP } \\
\hline & & Hyperplasia & WGP2 $=2$ & aGP2 & \\
\hline & \multirow{5}{*}{$\begin{array}{l}\text { Supporting } \\
\text { tissue }\end{array}$} & Hypertrophy & WGP3 = 1 & aGP3 & \\
\hline & & Hyperplasia & WGP4 = 2 & aGP4 & \\
\hline \multirow[t]{3}{*}{ Inflammation } & & Exudate & WGI1 = 1 & aGI1 & \multirow[t]{3}{*}{ IG1 } \\
\hline & & Activation of RES & WGI2 = 1 & $\mathrm{aGI} 2$ & \\
\hline & & Infilteration & WGI3 = 2 & aGI3 & \\
\hline \multirow[t]{3}{*}{ Tumor } & & Benign tumor & $\mathrm{WGT} 1=2$ & aGT1 & \multirow[t]{2}{*}{ IGT } \\
\hline & & Malignant tumor & WGT2 $=3$ & aGT2 & \\
\hline & & & & & IG \\
\hline \multirow{3}{*}{\multicolumn{2}{|c|}{$\begin{array}{l}\text { Liver } \\
\text { Circulatory } \\
\text { disturbances }\end{array}$}} & Hemorrhage/hyperemia & WLC $1=1$ & aLC1 & \multirow[t]{4}{*}{ ILC } \\
\hline & & /aneurysm & & & \\
\hline & & Intercellular edema & WLC2 = 1 & aLC2 & \\
\hline \multirow{4}{*}{$\begin{array}{l}\text { Regressive } \\
\text { changes }\end{array}$} & & & & & \\
\hline & Liver tissue & $\begin{array}{l}\text { Architectural and } \\
\text { structural alterations }\end{array}$ & WLR1 = 1 & aLR1 & \multirow[t]{3}{*}{ ILR } \\
\hline & & Plasma alterations & WLR2 = 1 & aLR2 & \\
\hline & & Deposits & WLR3 = 1 & aLR3 & \\
\hline
\end{tabular}


Table 2. Continued..

\begin{tabular}{|c|c|c|c|c|c|}
\hline & & Nuclear alterations & WLR4 = 2 & aLR4 & \\
\hline & & Atrophy & WLR5 = 2 & aLR5 & \\
\hline & & Necrosis & WLR6 = 3 & aLR6 & \\
\hline & & Vacuolar degeneration & & & \\
\hline & Interstitial tisste & $\begin{array}{l}\text { Architectural and } \\
\text { structural alterations }\end{array}$ & WLR7 = 1 & aLR7 & \\
\hline & & Plasma alterations & WLR8 = 1 & aLR8 & \\
\hline & & Deposits & WLR9 $=1$ & aLR9 & \\
\hline & & Nuclear alterations & WLR10 $=2$ & aLR10 & \\
\hline & & Atrophy & WLR11 = 2 & aLR11 & \\
\hline & & Necrosis & WLR12 $=3$ & aLR12 & \\
\hline & Bile duct & Architectural and & & & \\
\hline & & structural alterations & WLR13 = 1 & aLR13 & \\
\hline & & Plasma alterations & WLR14 = 1 & aLR14 & \\
\hline & & Deposits & WLR15 = 1 & aLR15 & \\
\hline & & Nuclear alterations & WLR16 = 2 & aLR16 & \\
\hline & & Atrophy & WLR17 = 2 & aLR17 & \\
\hline & & Necrosis & WLR18 $=3$ & aLR18 & \\
\hline Progressive & & & & & \\
\hline changes & Liver tissue & Hypertrophy & WLP1 = 1 & aLP1 & ILP \\
\hline & & Hyperplasia & WLP2 $=2$ & aLP2 & \\
\hline & Interstitial tissue & Hypertrophy & $\mathrm{WLP} 3=1$ & aLP3 & \\
\hline & & Hyperplasia & WLP4 = 2 & aLP4 & \\
\hline & Bile dudet & Hypertrophy & WLP5 $=1$ & aLP5 & \\
\hline & & Hyperplasia & WLP6 $=2$ & aLP6 & \\
\hline & & Wall proliferation of bil & ducts or duct & ules & \\
\hline Inflammation & & Exudate & WLI1 = 1 & aLI1 & IL1 \\
\hline & & Activation of RES & WLI2 = 1 & aLI2 & \\
\hline & & Infilteration & $\mathrm{WLI} 3=2$ & aLI3 & \\
\hline Tumor & & Benign tumor & WLT1 = 2 & aLT1 & ILT \\
\hline & & Malignant tumor & WLT2 $=3$ & aLT2 & \\
\hline & & & & & IL \\
\hline Kidney & & & & & \\
\hline Circulatory & & Hemorrhage/hyperemia & & & \\
\hline disturbances & & /aneurysm & $\mathrm{WKC} 1=1$ & $\mathrm{aKC} 1$ & IKC \\
\hline & & Intercellular edema & $\mathrm{WKC} 2=1$ & $\mathrm{aKC} 2$ & \\
\hline
\end{tabular}


Table 2. Continued..

\begin{tabular}{|c|c|c|c|c|c|}
\hline \multicolumn{6}{|l|}{ Regressive } \\
\hline \multirow[t]{21}{*}{ changes } & \multirow[t]{7}{*}{ Tubule } & Architectural and & & & \multirow{7}{*}{ IKR } \\
\hline & & structural alterations & WKR1 = 1 & aKR1 & \\
\hline & & Plasma alterations & $\mathrm{WKR} 2=1$ & aKR2 & \\
\hline & & Deposits & WKR3 = 1 & aKR3 & \\
\hline & & Nuclear alterations & WKR4 = 2 & aKR4 & \\
\hline & & Atrophy & WKR5 = 2 & aKR5 & \\
\hline & & Necrosis & WKR6 = 3 & aKR6 & \\
\hline & \multirow[t]{7}{*}{ Glomerulus } & \multirow{2}{*}{\multicolumn{4}{|c|}{$\begin{array}{l}\text { Architectural and } \\
\text { structural alterations }\end{array}$}} \\
\hline & & & & & \\
\hline & & Plasma alterations & WKR8 = 1 & aKR8 & \\
\hline & & Deposits & WKR9 = 1 & aKR9 & \\
\hline & & Nuclear alterations & WKR10 $=2$ & aKR10 & \\
\hline & & Atrophy & WKR11 = 2 & aKR11 & \\
\hline & & Necrosis & WKR12 = 3 & aKR12 & \\
\hline & \multicolumn{5}{|c|}{ Interstitial tissue Architectural and } \\
\hline & & structural alterations & WKR13 = 1 & aKR13 & \\
\hline & & Plasma alterations & WKR14 = 1 & aKR14 & \\
\hline & & Deposits & WKR15 = 1 & aKR15 & \\
\hline & & Nuclear alterations & WKR16 = 2 & aKR16 & \\
\hline & & Atrophy & WKR17 = 2 & aKR17 & \\
\hline & & Necrosis & WKR18 $=3$ & aKR18 & \\
\hline \multicolumn{6}{|l|}{ Progressive } \\
\hline \multirow[t]{8}{*}{ changes } & \multirow[t]{2}{*}{ Tubule } & Hypertrophy & WKP1 = 1 & aKP1 & IKP \\
\hline & & Hyperplasia & $\mathrm{WKP} 2=2$ & aKP2 & \\
\hline & \multirow[t]{4}{*}{ Glomerulus } & Hypertrophy & $\mathrm{WKP} 3=1$ & aKP3 & \\
\hline & & Hyperplasia & WKP4 = 2 & aKP4 & \\
\hline & & Thickening of & & & \\
\hline & & Bowman's capsular n & ane & & \\
\hline & \multirow[t]{2}{*}{ Interstitial tissıe } & Hypertrophy & WKP5 = 1 & aKP5 & \\
\hline & & Hyperplasia & WKP6 = 2 & aKP6 & \\
\hline \multirow[t]{3}{*}{ Inflammation } & & Exudate & $\mathrm{WKI} 1=1$ & aKI1 & IK1 \\
\hline & & Activation of RES & $\mathrm{WKI} 2=1$ & aKI2 & \\
\hline & & Infilteration & $\mathrm{WKI} 3=2$ & aKI3 & \\
\hline \multirow[t]{2}{*}{ Tumor } & & Benign tumor & $\mathrm{WKT} 1=2$ & aKT1 & IKT \\
\hline & & Malignant tumor & $\mathrm{WKT} 2=3$ & aKT2 & \\
\hline
\end{tabular}




\section{Mathematical calculation of lesion indices:}

\section{Reaction index of an organ ( $I_{\text {org }}$ rp $)$}

Only the lesions within one organ are studied, the following indices are applicable.

$$
I_{\text {org rp }}=\sum_{\text {alt }}\left(a_{\text {org rp alt }} \mathrm{x} w_{\text {org rp alt }}\right)
$$

where org $=$ organ; $r p=$ reaction pattern $($ constant $) ;$ alt $=$ alteration; $\mathrm{a}=$ score value; $\mathrm{w}=$ importance factor. The quality of the lesion in an organ is expressed by the reaction index.

\section{Organ index $\left(I_{\text {org }}\right)$}

$$
I_{\text {org }}=\sum_{\text {rp }} \sum_{\text {alt }}(a \text { org rp alt } \mathrm{X} w \text { org rp alt })
$$

(Abbreviations same as in reaction index formula). This index represents the degree of damage to an organ

\section{Total index (Tot-I)}

$$
\text { Tot }-I=\sum_{\text {org }} \sum_{\text {rp }} \sum_{\text {alt }}(a \text { org rp alt } \mathrm{X} w \text { org rp alt })
$$

(Abbreviations same as in reaction index formula). This index represents a measure of the overall health status based on the histological lesions.

\section{Results}

The organ index calculated based on various reaction patterns of the different organs showed that gills were severely affected, liver was moderately affected, and kidney was the mildly affected organ, irrespective of fish species (Table 3-13).

Fishes of same species collected from Kuttanad also showed similar pattern except for A. testudineus in which liver was less damaged than kidney (Table 10-11). The total index indicated the overall health status of the fishes in each concentration and in Kuttanad. There was a gradual decrease in the health status of fish according to the increase in concentration of pesticide 
Table 3. Organ index values of the gills of E. maculatus exposed to monocrotophos (following Bernet et al. 1999)

\begin{tabular}{|c|c|c|c|c|c|c|c|c|c|c|c|c|c|c|c|c|c|c|c|c|c|c|c|c|c|c|c|c|c|c|}
\hline \multirow{2}{*}{$\begin{array}{l}\begin{array}{l}\text { Concentra- } \\
\text { tions }\end{array} \\
\text { No. of } \\
\text { fishes } \\
\end{array}$} & \multicolumn{5}{|c|}{$0.0 \mathrm{ppm}$} & \multicolumn{5}{|c|}{$0.1 \mathrm{ppm}$} & \multicolumn{5}{|c|}{$0.3 \mathrm{ppm}$} & \multicolumn{5}{|c|}{$0.6 \mathrm{ppm}$} & \multicolumn{5}{|c|}{$1.0 \mathrm{ppm}$} & \multicolumn{5}{|c|}{$1.5 \mathrm{ppm}$} \\
\hline & 1 & 2 & 3 & 4 & 5 & 1 & 2 & 3 & 4 & 5 & 1 & 2 & 3 & 4 & 5 & 1 & 2 & 3 & 4 & 5 & 1 & 2 & 3 & 4 & 5 & 1 & 2 & 3 & 4 & 5 \\
\hline \multicolumn{31}{|l|}{ Alterations } \\
\hline WGCl=1 & . & . & . & . & . & . & . & . & . & . & . & . & . & . & . & $4 / 4$ & . & . & $4 / 4$ & $2 / 2$ & $2 / 2$ & $4 / 4$ & $4 / 4$ & $2 / 2$ & $4 / 4$ & $6 / 6$ & $6 / 6$ & $4 / 4$ & $4 / 4$ & $4 / 4$ \\
\hline WGC2 $=1$ & . & . & - & . & . & $6 / 6$ & $4 / 4$ & $2 / 2$ & $2 / 2$ & - & $4 / 4$ & $2 / 2$ & $4 / 4$ & $4 / 4$ & $2 / 2$ & $2 / 2$ & $4 / 4$ & $4 / 4$ & $2 / 2$ & $4 / 4$ & $4 / 4$ & $2 / 2$ & $4 / 4$ & $4 / 4$ & $2 / 2$ & $4 / 4$ & $4 / 4$ & $4 / 4$ & $4 / 4$ & $4 / 4$ \\
\hline WGR $2=1$ & . & . & . & . & . & $2 / 2$ & $2 / 2$ & $2 / 2$ & $2 / 2$ & $2 / 2$ & $2 / 2$ & $2 / 2$ & $2 / 2$ & $2 / 2$ & $2 / 2$ & . & . & . & . & . & $4 / 4$ & $2 / 2$ & $2 / 2$ & $2 / 2$ & $2 / 2$ & $4 / 4$ & $2 / 2$ & $2 / 2$ & $2 / 2$ & $2 / 2$ \\
\hline WGR $5=2$ & . & . & . & . & . & . & $8 / 4$ & $4 / 2$ & $8 / 4$ & 2. & $8 / 4$ & $4 / 2$ & $4 / 2$ & $4 / 2$ & . & $8 / 4$ & $8 / 4$ & $4 / 2$ & $4 / 2$ & . & & & & & $2 / 2$ & $\begin{array}{l}72 / 6 \\
12 / 6\end{array}$ & $8 / 4$ & $8 / 4$ & $8 / 4$ & $4 / 2$ \\
\hline WGR6 63 & . & . & . & . & . & . & . & . & - & . & - & . & - & . & . & - & - & . & - & . & $12 / 4$ & $6 / 2$ & $6 / 2$ & $12 / 4$ & - & $12 / 4$ & $12 / 4$ & $12 / 4$ & $12 / 4$ & $12 / 4$ \\
\hline WGR7 $=1$ & . & . & . & . & . & . & $2 / 2$ & . & . & . & $2 / 2$ & . & . & . & . & $4 / 4$ & . & $2 / 2$ & $2 / 2$ & $2 / 2$ & $6 / 6$ & $4 / 4$ & $4 / 4$ & $6 / 6$ & $4 / 4$ & $6 / 6$ & $4 / 4$ & $6 / 6$ & $4 / 4$ & $6 / 6$ \\
\hline WGR11=2 & & & & . & . & $8 / 4$ & $4 / 2$ & $4 / 2$ & . & . & $4 / 2$ & $8 / 4$ & $4 / 2$ & . & $8 / 4$ & $12 / 6$ & $8 / 4$ & $4 / 2$ & $8 / 4$ & $4 / 2$ & $12 / 6$ & $12 / 6$ & $8 / 4$ & $8 / 4$ & $12 / 6$ & $8 / 4$ & $12 / 6$ & $12 / 6$ & $8 / 4$ & $8 / 4$ \\
\hline WGPl $=1$ & $2 / 2$ & $2 / 2$ & . & . & $2 / 2$ & $2 / 2$ & $2 / 2$ & $2 / 2$ & $2 / 2$ & $2 / 2$ & $2 / 2$ & $2 / 2$ & $4 / 2$ & : & $2 / 2$ & . &. & $4 / 2$ & $8 / 4$ & $4 / 2$ & 120 & 120 & 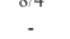 & & 120 & $8 / 4$ & . & 1270 & 0.14 &. \\
\hline WGP2 $=2$ & $4 / 2$ & . & $4 / 2$ & . & . & $4 / 2$ & $8 / 4$ & $4 / 2$ & $4 / 2$ & $8 / 4$ & $8 / 4$ & $12 / 6$ & $12 / 6$ & $4 / 2$ & $4 / 2$ & $4 / 2$ & $4 / 2$ & $8 / 4$ & $8 / 4$ & $4 / 2$ & $12 / 6$ & $8 / 4$ & $8 / 4$ & $12 / 6$ & $8 / 4$ & $4 / 2$ & $8 / 4$ & $8 / 4$ & $4 / 2$ & $4 / 2$ \\
\hline WGP4 $=2$ & . & . & $\cdot$ & - & . & & . & $\cdot$ & - & - & $12 / 6$ & $8 / 4$ & $4 / 2$ & $4 / 2$ & $8 / 4$ & $12 / 6$ & $8 / 4$ & $4 / 2$ & $4 / 2$ & $8 / 4$ & $8 / 4$ & $8 / 4$ & $4 / 2$ & $4 / 2$ & $4 / 2$ & $8 / 4$ & $12 / 6$ & $8 / 4$ & $12 / 6$ & $12 / 6$ \\
\hline WGTl $=2$ & . & . & . & . & . & . & . & . & . & . & - & - & - &. & - & - & - &. & - & - &. & - & - & & - & $4 / 2$ & - & - & - & - \\
\hline $\begin{array}{l}\text { Organ } \\
\text { index of } \\
\text { each fish }\end{array}$ & 6 & 2 & 4 & - & 2 & 22 & 30 & 18 & 18 & 12 & 42 & 38 & 30 & 18 & 26 & 46 & 32 & 26 & 32 & 24 & 60 & 46 & 40 & 50 & 36 & 68 & 68 & 64 & 58 & 56 \\
\hline $\begin{array}{l}\text { Mean organ } \\
\text { index of } 5 \\
\text { fishes }\end{array}$ & & & 2.8 & & & & & 20.0 & & & & & 30.8 & & & & & 32.0 & & & & & 46.4 & & & & & 62.8 & & \\
\hline
\end{tabular}

Denominator value denotes the score value: Numerator value $=$ (score value $\mathrm{x}$ importance factor) WGC $1=1$ means importance factor $=1$. 
Table 4. Organ index values of the liver of E. maculatus exposed to monocrotophos (following Bernet et al. 1999)

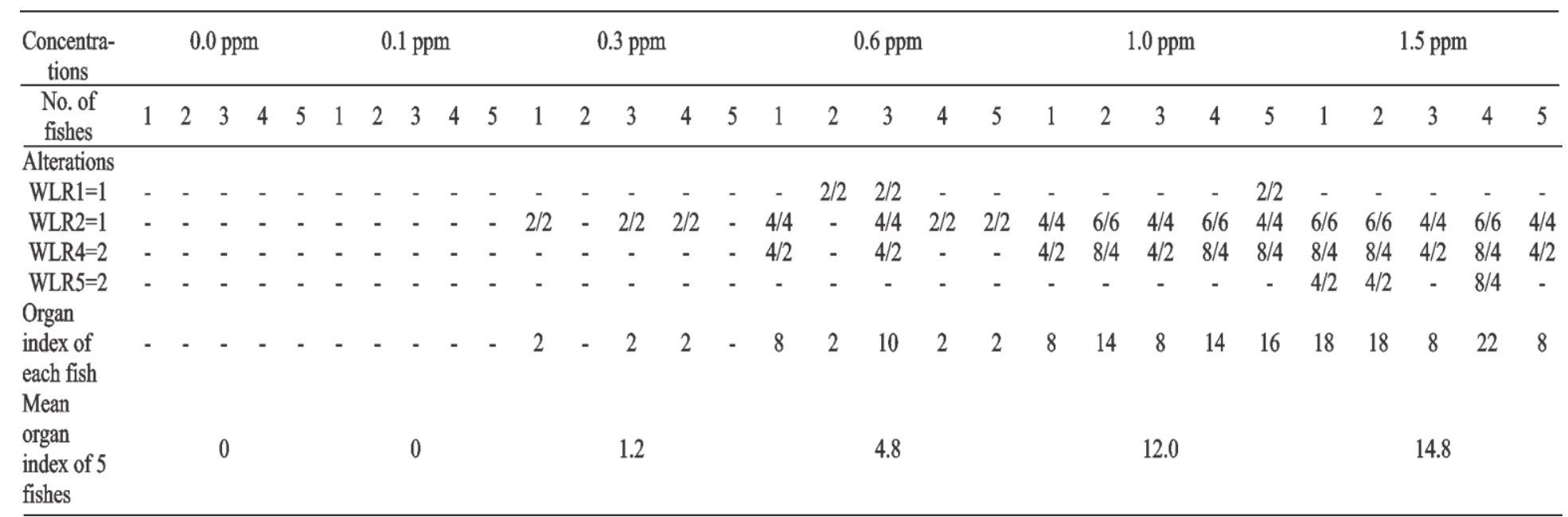

Denominator value denotes the score value: Numerator value $=($ score value $\mathrm{x}$ importance factor $)$. WLC $1=1$ means importance factor $=1$. 
Table 5. Organ index values of the liver of A. testudinues exposed to monocrotophos (following Bernet et al., 1999)

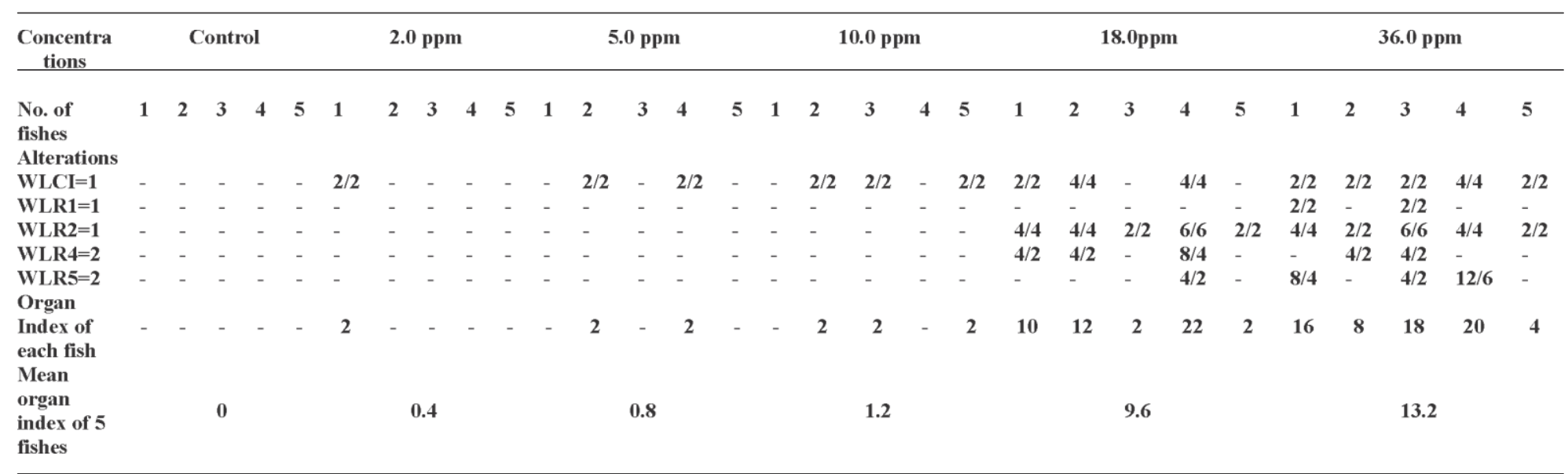

Denominator value denotes the score value: Numerator value $=($ score value $\mathrm{x}$ importance factor $)$. WLC $1=1$ means importance factor $=1$ 
Table 6. Organ index values of the kidney of E. maculatus exposed to monocrotophos (following Bernet et al., 1999)

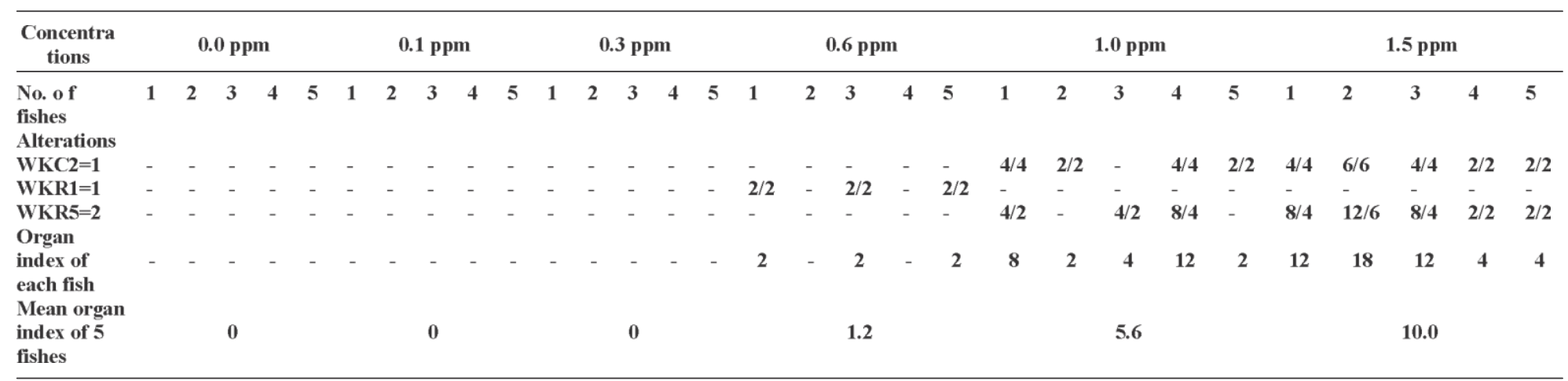

Denominator value denotes the score value: Numerator value $=$ (score value $\mathrm{x}$ importance factor). WKC $1=1$ means importance factor $=1$. 
Table 7. Organ index values of the kidney of A. testudineus exposed to monocrotophos (following Bernet et al., 1999)

\begin{tabular}{|c|c|c|c|c|c|c|c|c|c|c|c|c|c|c|c|c|c|c|c|c|c|c|c|c|c|c|c|c|c|c|}
\hline \multirow{2}{*}{$\begin{array}{c}\begin{array}{c}\text { Concentra } \\
\text { tions }\end{array} \\
\begin{array}{l}\text { No. of } \\
\text { fishes }\end{array}\end{array}$} & \multicolumn{5}{|c|}{$0.0 \mathrm{ppm}$} & \multicolumn{5}{|c|}{$2.0 \mathrm{ppm}$} & \multicolumn{5}{|c|}{$5.0 \mathrm{ppm}$} & \multicolumn{5}{|c|}{$10.0 \mathrm{ppm}$} & \multicolumn{5}{|c|}{$18.0 \mathrm{ppm}$} & \multicolumn{5}{|c|}{$36.0 \mathrm{ppm}$} \\
\hline & 1 & 2 & 3 & 4 & 5 & 1 & 2 & 3 & 4 & 5 & 1 & 2 & 3 & 4 & 5 & 1 & 2 & 3 & 4 & 5 & 1 & 2 & 3 & 4 & 5 & 1 & 2 & 3 & 4 & 5 \\
\hline Alterations & & & & & & & & & & & & & & & & & & & & & & & & & & & & & & \\
\hline WKC2=1 & - & - & - & - & - & - & & - & - & - & - & - & - & - & - & $2 / 2$ & - & $4 / 4$ & - & $2 / 2$ & $2 / 2$ & $4 / 4$ & $2 / 2$ & $4 / 4$ & $4 / 4$ & $6 / 6$ & $4 / 4$ & $6 / 6$ & $6 / 6$ & $2 / 2$ \\
\hline WKR5=2 & - & - & - & - & - & - & - & - & - & - & - & - & - & - & - & $4 / 2$ & - & $4 / 2$ & - & - & - & - & - & - & - & $8 / 4$ & $4 / 2$ & - & $4 / 2$ & - \\
\hline WKP2=2 & - & - & - & - & - & - & - & - & - & - & - & - & - & - & - & - & - & - & - & - & $4 / 2$ & $4 / 2$ & $4 / 2$ & $4 / 2$ & $4 / 2$ & $12 / 6$ & $8 / 4$ & $8 / 4$ & $8 / 4$ & $12 / 6$ \\
\hline Organ & & & & & & & & & & & & & & & & & & & & & & & & & & & & & & \\
\hline $\begin{array}{l}\text { index of } \\
\text { each fish }\end{array}$ & - & - & - & - & - & - & - & - & - & - & - & - & - & - & - & 6 & - & 8 & - & 2 & 6 & 8 & 6 & 8 & 8 & 26 & 16 & 14 & 18 & 14 \\
\hline $\begin{array}{l}\text { Mean } \\
\text { organ } \\
\text { index of } 5 \\
\text { fishes }\end{array}$ & & & $\mathbf{0}$ & & & & & 0 & & & & & $\mathbf{0}$ & & & & & 3.2 & & & & & 7.2 & & & & & 17.6 & & \\
\hline
\end{tabular}

Denominator value denotes the score value: Numerator value $=($ score value $\mathrm{x}$ importance factor $)$. WKC2 $=1$ means importance factor $=1$. 
Table 8. Organ index values of the gills of A. testudineus exposed to monocrotophos (following Bernet et al. 1999)

\begin{tabular}{|c|c|c|c|c|c|c|c|c|c|c|c|c|c|c|c|c|c|c|c|c|c|c|c|c|c|}
\hline \multirow{2}{*}{$\begin{array}{l}\begin{array}{c}\text { Concentra } \\
\text { tions }\end{array} \\
\begin{array}{l}\text { No. of } \\
\text { fishes }\end{array}\end{array}$} & \multicolumn{5}{|c|}{$0.0 \mathrm{ppm}$} & \multicolumn{5}{|c|}{$2.0 \mathrm{ppm}$} & \multicolumn{5}{|c|}{$5.0 \mathrm{ppm}$} & \multicolumn{5}{|c|}{$10.0 \mathrm{ppm}$} & \multicolumn{5}{|c|}{$18.0 \mathrm{ppm}$} \\
\hline & 1 & 2 & 3 & 4 & 5 & 1 & 2 & 3 & 4 & 5 & 1 & 2 & 3 & 4 & 5 & 1 & 2 & 3 & 4 & 5 & 1 & 2 & 3 & 4 & 5 \\
\hline $\mathrm{WGC2}=1$ & - & - & - & - & - & $2 / 2$ & $2 / 2$ & $2 / 2$ & - & $2 / 2$ & $2 / 2$ & $2 / 2$ & $4 / 4$ & $2 / 2$ & $4 / 4$ & $4 / 4$ & $2 / 2$ & $4 / 4$ & $2 / 2$ & $4 / 4$ & $6 / 6$ & $4 / 4$ & $2 / 2$ & $6 / 6$ & $4 / 4$ \\
\hline WGR1=1 & - & - & - & - & - & - & - & - & - & - & - & - & - & - & - & - & - & - & - & - & - & - & - & - & - \\
\hline WGR5 $=2$ & - & - & - & - & - & - & - & - & - & - & $4 / 2$ & $4 / 2$ & - & $4 / 2$ & $4 / 2$ & $4 / 2$ & $4 / 2$ & $4 / 2$ & $4 / 2$ & $4 / 2$ & $4 / 2$ & $4 / 2$ & $8 / 4$ & $4 / 2$ & $4 / 2$ \\
\hline WGR7 $=1$ & - & - & - & - & - & - & - & - & - & - & - & - & - & - & - & $2 / 2$ & $2 / 2$ & - & $4 / 4$ & $2 / 2$ & $4 / 4$ & $2 / 2$ & - & $2 / 2$ & - \\
\hline WGR11=2 & - & - & - & - & - & - & - & - & - & - & $8 / 4$ & $4 / 2$ & - & $4 / 2$ & $4 / 2$ & $8 / 4$ & $8 / 4$ & - & $4 / 2$ & $4 / 2$ & $8 / 4$ & $8 / 4$ & $12 / 6$ & $4 / 2$ & $4 / 2$ \\
\hline WGP1 $=1$ & $2 / 2$ & $2 / 2$ & - & - & - & $2 / 2$ & - & $2 / 2$ & $2 / 2$ & - & $6 / 6$ & $2 / 2$ & $2 / 2$ & $4 / 4$ & $2 / 2$ & $2 / 2$ & $4 / 4$ & $2 / 2$ & $2 / 2$ & $4 / 4$ & - & - & - & - & - \\
\hline WGP2 $=2$ & $4 / 2$ & - & - & $4 / 2$ & $4 / 2$ & $4 / 2$ & $4 / 2$ & - & - & $4 / 2$ & - & - & - & - & - & $8 / 4$ & $4 / 2$ & $4 / 2$ & $4 / 2$ & - & $8 / 4$ & $4 / 2$ & $4 / 2$ & $8 / 4$ & $4 / 2$ \\
\hline WGP4=2 & - & - & - & - & - & - & - & - & - & - & - & - & - & - & - & $2 / 2$ & - & $4 / 4$ & $2 / 2$ & $2 / 2$ & $4 / 4$ & $2 / 2$ & $2 / 2$ & - & $2 / 2$ \\
\hline $\begin{array}{l}\text { Organ } \\
\text { index of } \\
\text { each fish } \\
\text { Mean }\end{array}$ & 6 & 2 & - & 4 & 4 & 8 & 6 & 4 & 2 & 6 & 20 & 12 & 6 & 14 & 14 & 30 & 24 & 18 & 22 & 20 & 34 & 24 & 28 & 24 & 18 \\
\hline $\begin{array}{l}\text { organ } \\
\text { Index of } 5 \\
\text { fishes }\end{array}$ & & & 3.2 & & & & & 5.2 & & & & & 13.2 & & & & & 22.8 & & & & & 25.6 & & \\
\hline
\end{tabular}

Denominator value denotes the score value: Numerator value $=$ (score value $\mathrm{x}$ importance factor). $\mathrm{WGC} 1=1$ means importance factor $=1$. 
Table 9. Organ index values of the gills of E. maculatus and A. testudineus collected from the paddy fields of Kuttanad (following Bernet et al., 1999)

\begin{tabular}{|c|c|c|c|c|c|c|c|c|c|c|c|c|c|c|c|c|c|c|c|c|}
\hline \multirow{2}{*}{$\begin{array}{l}\text { Fish species } \\
\text { No. of fishes } \\
\text { Alterations }\end{array}$} & \multicolumn{10}{|c|}{ E. maculatus } & \multicolumn{10}{|c|}{ A. testudineus } \\
\hline & 1 & 2 & 3 & 4 & 5 & 6 & 7 & 8 & 9 & 10 & 1 & 2 & 3 & 4 & 5 & 6 & 7 & 8 & 9 & 10 \\
\hline WGC1=1 & $4 / 4$ & $2 / 2$ & $4 / 4$ & $6 / 6$ & $2 / 2$ & $2 / 2$ & $4 / 4$ & $4 / 4$ & $6 / 6$ & $4 / 4$ & $4 / 4$ & $2 / 2$ & - & $2 / 2$ & $4 / 4$ & - & $4 / 4$ & $4 / 4$ & $2 / 2$ & - \\
\hline WGC2 $=1$ & $4 / 4$ & $4 / 4$ & $2 / 2$ & $2 / 2$ & $4 / 4$ & $4 / 4$ & $2 / 2$ & $4 / 4$ & $2 / 2$ & $4 / 4$ & $6 / 6$ & $\mathbf{4} / \mathbf{4}$ & $6 / 6$ & $2 / 2$ & $6 / 6$ & $4 / 4$ & $4 / 4$ & $6 / 6$ & $4 / 4$ & $2 / 2$ \\
\hline WGR5=2 & $8 / 4$ & $8 / 4$ & $4 / 2$ & $4 / 2$ & $12 / 6$ & $8 / 4$ & $8 / 4$ & $4 / 2$ & $12 / 6$ & $12 / 6$ & $8 / 4$ & $12 / 6$ & $4 / 2$ & $12 / 6$ & - & $4 / 2$ & $8 / 4$ & $8 / 4$ & $12 / 6$ & - \\
\hline WGR6=3 & $12 / 4$ & $18 / 6$ & $12 / 4$ & $6 / 2$ & $6 / 2$ & $12 / 4$ & $12 / 4$ & $6 / 2$ & $12 / 4$ & $12 / 4$ & $12 / 4$ & $6 / 2$ & $6 / 2$ & - & $12 / 4$ & $12 / 4$ & $12 / 4$ & $6 / 2$ & $6 / 2$ & $6 / 2$ \\
\hline WGR7=1 & $6 / 6$ & $4 / 4$ & $4 / 4$ & $2 / 2$ & $4 / 4$ & $4 / 4$ & $4 / 4$ & $2 / 2$ & $6 / 6$ & $4 / 4$ & $6 / 6$ & $4 / 4$ & $6 / 6$ & $4 / 4$ & $4 / 4$ & $4 / 4$ & $2 / 2$ & $6 / 6$ & $2 / 2$ & $6 / 6$ \\
\hline WGR11=2 & $12 / 6$ & $4 / 2$ & $8 / 4$ & $4 / 2$ & $4 / 2$ & $8 / 4$ & $12 / 6$ & $12 / 6$ & $8 / 4$ & $4 / 2$ & $8 / 4$ & - & $4 / 2$ & $4 / 2$ & $8 / 4$ & $8 / 4$ & $4 / 2$ & $8 / 4$ & - & $8 / 4$ \\
\hline WGP2=2 & $12 / 6$ & $8 / 4$ & $8 / 4$ & $12 / 6$ & $8 / 4$ & $12 / 6$ & $12 / 6$ & $12 / 6$ & $8 / 4$ & $4 / 2$ & $8 / 4$ & $12 / 6$ & - & $4 / 2$ & $12 / 6$ & $12 / 6$ & $8 / 4$ & $4 / 2$ & $12 / 6$ & $8 / 4$ \\
\hline $\begin{array}{l}\text { Organ index } \\
\text { of each fish }\end{array}$ & 58 & 48 & 42 & 36 & 40 & 50 & 54 & 44 & 54 & 44 & 52 & 40 & 26 & 28 & 46 & 44 & 42 & 42 & 38 & 30 \\
\hline $\begin{array}{l}\text { Mean organ } \\
\text { index of } 10 \\
\text { fishes }\end{array}$ & \multicolumn{10}{|c|}{47.0} & \multicolumn{10}{|c|}{38.8} \\
\hline
\end{tabular}

Denominator value denotes the score value: Numerator value $=$ (score value $\mathrm{x}$ importance factor). WGC $1=1$ means importance factor $=1$. 
Table 10. Organ index values of the liver of E. maculatus and A. testudineus collected from the paddy fields of Kuttanad (following Bernet et al., 1999)

\begin{tabular}{|c|c|c|c|c|c|c|c|c|c|c|c|c|c|c|c|c|c|c|c|c|}
\hline \multirow{2}{*}{$\begin{array}{l}\text { Fish species } \\
\begin{array}{l}\text { No of fishes } \\
\text { Alterations }\end{array}\end{array}$} & \multicolumn{10}{|c|}{ E. maculatus } & \multicolumn{10}{|c|}{ A. testudeneus } \\
\hline & 1 & 2 & 3 & 4 & 5 & 6 & 7 & 8 & 9 & 10 & 1 & 2 & 3 & 4 & 5 & 6 & 7 & 8 & 9 & 10 \\
\hline WLC1=1 & $2 / 2$ & - & $2 / 2$ & - & - & - & $2 / 2$ & $2 / 2$ & $2 / 2$ & - & - & - & $2 / 2$ & $2 / 2$ & $2 / 2$ & $4 / 4$ & $4 / 4$ & - & $2 / 2$ & - \\
\hline WLR2=1 & $6 / 6$ & $4 / 4$ & $4 / 4$ & $2 / 2$ & $2 / 2$ & $4 / 4$ & $6 / 6$ & $6 / 6$ & $6 / 6$ & $2 / 2$ & - & - & - & - & - & - & - & - & - & - \\
\hline WLR4=2 & $8 / 4$ & $4 / 2$ & $4 / 2$ & - & - & $4 / 2$ & $8 / 4$ & $8 / 4$ & $8 / 4$ & - & $4 / 2$ & $8 / 4$ & $4 / 2$ & $4 / 2$ & $4 / 2$ & $8 / 4$ & $8 / 4$ & $4 / 2$ & $4 / 2$ & $8 / 4$ \\
\hline WLR5=2 & $4 / 2$ & - & - & $4 / 2$ & $4 / 2$ & - & $4 / 2$ & $4 / 2$ & - & $4 / 2$ & - & - & - & - & - & - & - & - & - & - \\
\hline WLR6 $=3$ & $6 / 2$ & - & - & - & - & - & $6 / 2$ & $6 / 2$ & $6 / 2$ & - & - & - & - & - & - & - & - & - & - & - \\
\hline WLT1=2 & - & - & - & - & - & - & - & - & - & - & $4 / 2$ & - & $4 / 2$ & - & - & $8 / 4$ & - & $8 / 4$ & - & - \\
\hline $\begin{array}{l}\text { Organ Index } \\
\text { of each fish } \\
\text { Mean organ }\end{array}$ & 26 & 8 & 10 & 6 & 6 & 8 & 26 & 26 & 22 & 6 & 8 & 8 & 10 & 6 & 6 & 20 & 12 & 12 & 6 & 8 \\
\hline $\begin{array}{l}\text { Index of } 10 \\
\text { fishes }\end{array}$ & \multicolumn{10}{|c|}{13.6} & \multicolumn{10}{|c|}{9.6} \\
\hline
\end{tabular}

Denominator value denotes the score value: Numerator value $=($ score value $\mathrm{x}$ importance factor $)$. WLC $1=1$ means importance factor $=1$. 
Table 11. Organ index values of the Kidney of E. maculatus and A. testudineus collected from the paddy fields of Kuttanad (following Bernet et al., 1999)

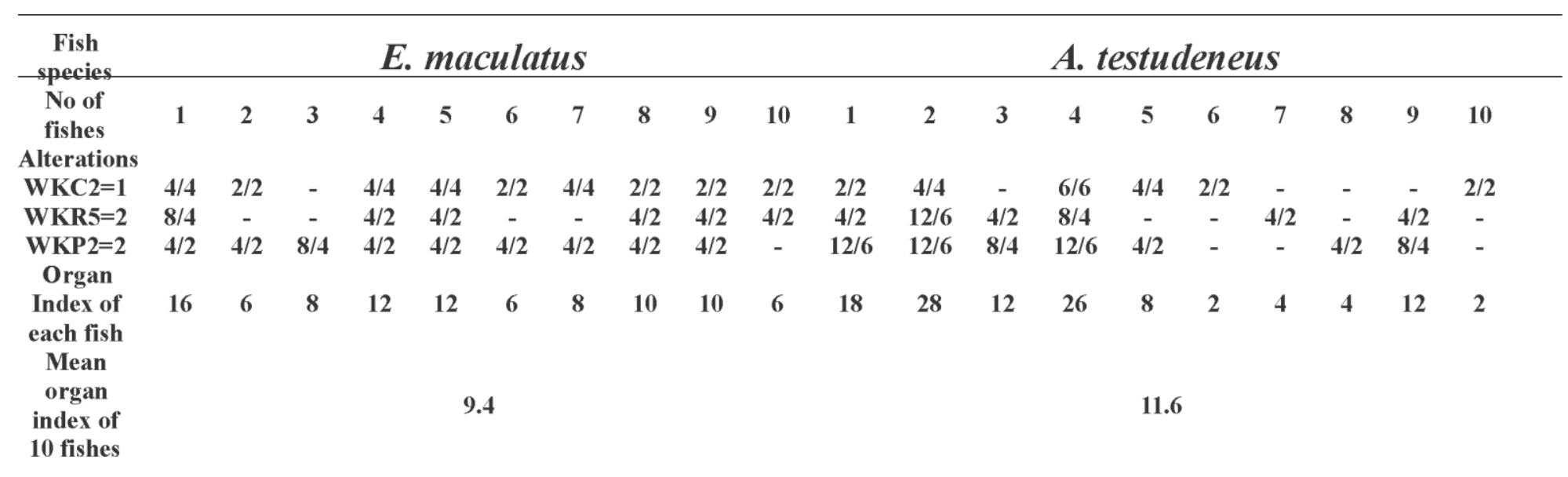

Denominator value denotes the score value: Numerator value $=$ (score value $\mathrm{x}$ importance factor). $\mathrm{W}_{\mathrm{KC} 2}=1$ means importance factor $=1$. 
Table 12. Total index of E. maculatus and A. testudineus exposed to different sublethal concentrations of monocrotophos based on the organ index.

\begin{tabular}{lccccc}
\hline \multirow{2}{*}{ Fish } & $\begin{array}{c}\text { Treatment } \\
(\mathrm{ppm})\end{array}$ & Gill & Organ Index & Kiver & Total Index \\
\cline { 2 - 5 } E. maculatus & 0.0 & 2.8 & 0 & 0 & 2.8 \\
& 0.1 & 20.0 & 0 & 0 & 20.0 \\
& 0.3 & 30.8 & 1.2 & 0 & 32.0 \\
& 0.6 & 32.0 & 1.8 & 1.2 & 38.0 \\
& 1.0 & 46.4 & 14.8 & 10.0 & 64.0 \\
& 1.5 & 62.8 & 0 & 0 & 87.6 \\
\hline \multirow{5}{*}{ A. testudineus } & 5.0 & 5.2 & 0.4 & 0 & 3.2 \\
& 10.0 & 13.2 & 0.8 & 0 & 5.6 \\
& 18.0 & 22.8 & 1.2 & 3.2 & 14.0 \\
& 36.0 & 25.6 & 9.6 & 7.2 & 27.2 \\
\hline
\end{tabular}

Table 13. Total index of E. maculatus and A. testudineus collected from Kuttanad based on the organ index

\begin{tabular}{ccccc}
\hline & \multicolumn{3}{c}{ Organ Index } & \multirow{2}{*}{ Total Index } \\
\cline { 2 - 4 } Fish Species & Gill & Li ver & Kidney & \\
\hline E. maculatus & 47.0 & 13.6 & 9.4 & 70.0 \\
A. testudineus & 38.8 & 9.6 & 11.6 & 60.0 \\
\hline
\end{tabular}




\section{Discussion}

The organ indices are used for calculating the total index, which gives the health status of an organism in particular, under altered environmental condition. In the present study, the total index showed the health status of fishes in each sublethal concentration and in the field conditions. The health status became worse in the higher sublethal concentrations of both the fishes treated with monocrotophos. The total index of $E$. maculatus collected from Kuttanad is 70 , which is comparable to the total index value of E. maculatus exposed to sublethal concentrations of $1.0 \mathrm{ppm}$ monocrotophos (total index 64). The total index value of A. testudineus collected from Kuttanad is 60 , which is comparable to the total index value of A. testudineus exposed to sublethal concentrations of $36 \mathrm{ppm}$ monocrotophos (total index 63.4).

It is evident from the index value that the gills are in the irreversibly damaged condition as per Poleksic \& Mitrovic-Tutundzic (1994). This irreversible condition in the gills of E. maculatus and A. testudineus may be due to the chronic microtoxicosis (sublethal effects) as a result of toxicants in the medium. Thus, gill degeneration can be considered as a factor, which seriously impairs the viability of organisms, while this may not represent a hazard to the life of the individual. It has great importance as far as the survival of the population is concerned. Szakolczai et al. (1994) have also reported that structural changes in gills can be considered suitable to monitor the level of environmental contamination, especially the sublethal and chronic effects of pollutants, particularly in those cases where other methods of assessments are not satisfactory, and to compliment the assessment of the average level of pollution. It should be emphasized that fish gill can maintain their vital functions even when some lamellae are heavily damaged. However, chronic exposure of these gill lamellae to pesticides will lead to the histological degeneration of the irreversible condition that will lead to functional disturbance or dysfunction of the organ. This gradually leads to mortality and in turn affects the population of the ecosystem. Hence, the present study carried out on the natural population supports the view of Poleksic \& Mitrovic-Tutundzic (1994) that histological changes in fish gills should become one of the methods used for assessment of water quality in sublethal and chronic situations.

The histopathological changes are one of the most sensitive parameters for the evaluation of chronic toxicity test effects and thus also for the derivation of Maximum Allowable Toxicant Concentration as reported by Poleksic \& Mitrovic-Tutundzic (1994). Moreover, the sublethal concentrations may become lethal for populations confronted 
with additional stresses. This should be taken into serious consideration when evaluating the effects of mixtures of toxicants in fresh water fish under natural conditions.

In the natural ecosystem, fishes are exposed simultaneously to more than one biocide or contaminant because some chemicals are applied continuously and are highly persistent or others are applied as combinations to increase the efficiency or reduce costs (Marking 1977). Kuttanad is an area where the pesticides and weedicides are applied simultaneously or intermittently. The problems of toxicity of mixtures of pesticides on fish have been recognized only recently and notable among the studies are those of Macek (1977); Fabacher et al. (1976); Nair et al. (2000). While studying the individual and combined acute lethal toxicity of monocrotophos and 2,4-D on the juveniles of E. suratensis, a highly favored food fish of Kuttanad region, Nair et al. (2000) reported that a strictly additive nature of their combined toxicity and the sequential and even simultaneous use in the ecosystem increases the potential for pollution. Significant increase in sensitivity could be achieved from histological studies when compared with routine parameters like survival and mortality. Histopathology provides evidences of adaptation to degeneration, and this certainly represents the major advantage of the use of histopathological alterations as biomarkers of environmental pollution by organic chemicals.

Therefore, it is proposed that the histological changes in fish gill should become one of the methods used for assessment of water quality in sublethal and chronic situations as the toxicants induce changes at lower levels of biological organization occurring prior to organismic changes. It should therefore provide a rapid "early warning system" as suggested by Moore (1985).

\section{Acknowledgement}

The Science, Technology, Environment Committee, Kerala State (STEC), which supported this study financially, is gratefully acknowledged. The authors are grateful to the Dean, College of Fisheries, Kochi for providing facilities to carry out the work.

\section{References}

Adams, S.M., K.I. Shepard, M.S.Greeley, B.D .Jr. Jimenez, M.G. Ryon, L.R. Shugart and J.F. McCarthy. 1989. The use of bioindicators for assessing the effects of pollutants stress on fish. Marine Environmental Research $28: 459-464$

Bernet, D., H. Schmidt, W.Meir, P. Burkhardt-Holn and T. Wahli. 1999. Histopathology in fish: Proposal for a protocol to assess aquatic pollution. Journal of Fish Diseases 22:25-34.

Fabacher, D.L., J.D. Davis and D.A. Fabacher. 1976. Apparent Potentiation of the Cotton defoliant DEF by methylparathion in mosquitofish. Bulletin of Environmental Contamination and Toxicology 16:716.

Hinton, D.E. 1993. Toxicologic histopathology of fishes: A systematic approach and overview. In: Pathobiology of marine and estuarine organisms. (ed. John A. Couch and John W. Fourine), pp. 117-215. CRC Press, Boca Raton Ann Arobor, London, Tokyo. 
John, K.R., N. Jayabalan and M.R.George.1993. Impact of sub-lethal concentrations of endosulfan on the histology of Cyprinius carpio liver and kidney. Proceedings of the National Seminar on Aquaculture Development in India Problems and Prospects, 27-29: November 1990 (ed. P. Natarajan and V. Jayaprakas), pp. 179182. Thiruvananthapuram, India, Kerala.

Kurup, B.M., M.J. Sebastian, T.M. Sankaran and P. Rabindranath. 1990. Exploited fishery resources of the Vembanad lake. Final report presented to Indo-Dutch Co-operation programme. pp144.

Konar, S.K. 1969. Two organophosphorus insecticides DDVP and phosphamidon as selective toxicants. Transactions of the American Fisheries Society 98:430-437.

KWBSP.1990. Report of Kuttanad water balance study project. Indo-Dutch Co-operation programme by college of Fisheries, Panangad.

Macek, K.J. and B.H. Sleight. 1977. Utility of toxicity tests with embryos and fry of fish in evaluating hazards associated with the chronic toxicity of chemicals to fishes. In: Aquatic toxicology and hazard evaluation (ed. F.L. Mayer and J.L. Hamelink), pp. 137-146. ASTM STP 634. Amercian Society for Testing and Materials, Philadelphia,.

Marking, L.L. 1977. Method for assessing additive toxicity of chemical mixtures. In: Aquatic toxicology and hazard Evaluation (ed. F.L. Mayer and J.L. Hamelink), pp. 99-108 ASTM STP 634, American Society for Testing of Materials, Philadelphia.

Mercy, T.V.A., B. Madhusoodana Kurup, J.R.Nair and B.T. Sulekha 2000. Lethal toxicity of monocrotophos on the juveniles of Anabas testudineus (Bloch) and Etroplus maculatus (Bloch). Indian Journal of Fisheries 47(3):253-256.

Moore, M.N. 1985. Cellular responses to pollutants. Marine Pollution Bulletin 16:134-139.

Nair, J.R., T.V.A. Mercy and Renu Maria George. 2000. Individual and combined lethal toxicity of monocrotophos and 2,4-D on the juveniles of Etroplus suratensis (Bloch) (Pisces-Cichlidae). Fishery Technology 37 (2):116-120.

Poleksic, V. and V. Mitrovic-Tutundzic 1994. Fish gills as a monitor of sublethal and chronic effects of pollution. In: Sublethal and chronic effects of pollution on freshwater fish (ed. R. Muller and R. Llyod), pp. 339-352. FAO Fishing News Books, Oxford, UK.

Sprague, J.B. 1973. The ABC's pollutant bioassay using fish. In: Biological methods for the assessment of water quality. (ed. J. Cairns, Jr. and K.L. Dickson), pp. 6-30 ASTM STP 528, American Society for testing and Materials, Philadelphia.

Szakolczai, J., J. Ramotsa, M. Miklovics and G. Csaba. 1994. Monitoring system for investigation of heavy metal and chlorinated hydrocarbon pollution of fish in natural waters and fish ponds. In: Sublethal and chronic effects of pollutant on freshwater fish (ed. R. Muller and R.Lloyd), Pp. 359-364. FAO, Fishing News Books, Oxford, UK. 